\title{
Choice of sedation in endoscopic retrograde cholangiopancreatography: is monitored anesthesia care as safe as general anesthesia? A systematic review and meta-analysis
}

\author{
Amaninder Dhaliwala , Banreet Singh Dhindsab ${ }^{b}$ Syed Mohsin Saghirc, Daryl Ramaid, Saurabh Chandane,

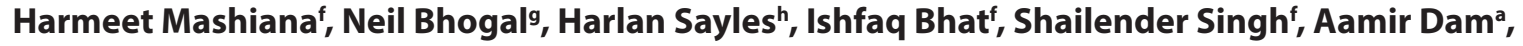 \\ Pushpak Taunk', Rene Gomez Esquiveli, Jason Klapmana, Stephanie McDonough', Douglas G. Adlerj \\ Moffitt Cancer Center, Tampa, FL; University of Nebraska Medical Center, Omaha, NE; University of Nevada Las \\ Vegas School of Medicine, Las Vegas; The Brooklyn Hospital Center, Brooklyn, NY; Creighton University School of \\ Medicine, Omaha, NE; University of South Florida Morsani College of Medicine, Tampa, FL; University of Utah School \\ of Medicine, Salt Lake City, UT, USA
}

\section{Abstract}

\begin{abstract}
Background Monitored anesthesia care (MAC) and general anesthesia (GA) are the 2 most common methods of sedation used for endoscopic retrograde cholangiopancreatography (ERCP). We performed a systematic review and meta-analysis to compare the overall safety between MAC vs. GA in ERCP.
\end{abstract}

Methods We conducted a comprehensive search of electronic databases to identify studies reporting the use of MAC or GA as a choice of sedation for ERCP. The primary outcome was to compare the overall rate of sedation-related adverse events in MAC vs. GA groups. The secondary endpoint was to investigate the total duration of the procedure, recovery time, ERCP cannulation rates, and conversion rate of MAC to GA. The meta-analysis was performed using a Der Simonian and Laird random-effects model.

Results A total of 21 studies reporting on 11,592 patients were included. The overall sedationrelated side-effects were similar in the GA $(12.76 \%$, 95\% confidence interval [CI] 5.80-21.73; $\left.I^{2}=95 \%\right)$ and MAC $\left(12.08 \%, 95 \%\right.$ CI 5.38-20.89; $\left.I^{2}=99 \%\right)$ groups $(\mathrm{P}=0.956)$. Hypoxia, arrhythmias, hypotension, aspiration and other sedation-related side-effects were similar between the 2 groups. The mean duration of the procedure was longer in the MAC group, but the mean recovery time was shorter. Significant heterogeneity was noted in our meta-analysis.

Conclusions In our meta-analysis, the overall sedation-related side-effects were similar between the MAC and GA groups. MAC could be used as a safer alternative to GA when performing ERCP. However, large multicenter randomized control trials are needed to further validate our findings.

Keywords Sedation, ERCP, anesthesia, adverse events, meta-analysis

Ann Gastroenterol 2021; 34 (x): 1-15

\section{Introduction}

Endoscopic retrograde cholangiopancreatography (ERCP) is the treatment of choice in pancreaticobiliary diseases [1]. The

Conflict of Interest: None

Correspondence to: Douglas G. Adler, MD, FACG, AGAF, FASGE, Professor of Medicine, Director of Therapeutic endoscopy, Director, GI fellowship program, Gastroenterology and Hepatology, University of Utah School of Medicine, Huntsman Cancer Center, 30 N 1900 E, Room 4R118, Salt Lake City, Utah 84132, USA, e-mail: dougraham2001@gmail.com

Received 21 December 2020; accepted 20 April 2021; published online 2 July 2021

DOI: https://doi.org/10.20524/aog.2021.0650
2 types of anesthesia used for ERCP at most institutions are monitored anesthesia care (MAC) or general anesthesia (GA). MAC involves an additional anesthesia provider employed to control the level of patients' sedation, pain and anxiety, while preserving their spontaneous breathing and intact airway reflexes. Patients should be able to respond to verbal commands, maintain their airway, and spontaneously ventilate. Often a combination of sedatives is employed to achieve a rapid onset, high clearance of drugs and minimal side effects [2,3]. GA is similar to MAC in that it also utilizes multiple sedatives to control patients' sedation, pain and anxiety, while also dampening their autonomic nervous system and paralyzing their skeletal muscles. Patients are unarousable to stimulation, are in deep sedation, and are unable to maintain a patent airway or their own ventilation independently. Patients undergoing ERCP via GA also have 
their airway accessed and protected via an endotracheal tube [4]. There remains an ongoing debate regarding the relative merits and risks of MAC vs. GA for patients undergoing ERCP.

This systematic review and meta-analysis compares the overall safety of MAC vs. GA for patients undergoing ERCP. The primary endpoint is to compare the adverse events of both types of anesthesia used in ERCP procedures. The secondary endpoint is to investigate the total duration of the procedure, recovery time, ERCP cannulation rates, and conversion rate of MAC to GA.

\section{Materials and methods}

\section{Search strategy}

We conducted a comprehensive search of multiple databases and conference proceedings, including PubMed, EMBASE, Google-Scholar, LILACS, Scopus, and Web of Science (inception to 3/2020). The "Preferred Reporting Items for Systematic reviews and Meta-Analyses" (PRISMA) guidelines were used to identify studies reporting on outcomes among patients undergoing ERCP with sedation via MAC and/or GA [5]. The literature search was performed by an experienced medical librarian using inputs from the study authors.

Key words used in the literature search included a combination of "ERCP, "MAC, "general”, "anesthesia, "sedation", "monitored", "adverse events", "hypoxia" and "cannulation rates". The search was restricted to studies in human subjects published in the English language in peer-reviewed journals. Two authors (BD, JS) independently reviewed the titles and abstracts of studies identified in the primary search and excluded studies that did not address the research question, based on pre-specified exclusion and inclusion criteria. The full text of remaining articles was reviewed to determine whether they contained relevant information. Any discrepancy in article selection was resolved by consensus, and in discussion with a

${ }^{a}$ Division of Gastroenterology and Hepatology, Moffitt Cancer Center, Tampa, FL (Amaninder Dhaliwal, Aamir Dam, Jason Klapman); ${ }^{b}$ Department of Internal Medicine, Division of Gastroenterology and Hepatology, University of Nebraska Medical Center, Omaha, NE (Banreet Singh Dhindsa); 'Department of Internal Medicine, University of Nevada Las Vegas School of Medicine, Las Vegas (Syed Mohsin Saghir); ${ }^{\mathrm{d} D e p a r t m e n t ~ o f ~ I n t e r n a l ~ M e d i c i n e, ~ T h e ~}$ Brooklyn Hospital Center, Brooklyn, New York (Daryl Ramai); ${ }^{\mathrm{e}}$ Division of Gastroenterology and Hepatology, Creighton University School of Medicine, Omaha, NE (Saurabh Chandan); 'Division of Gastroenterology and Hepatology, University of Nebraska Medical Center, Omaha, NE (Harmeet Mashiana, Ishfaq Bhat, Shailender Singh); ${ }^{\mathrm{a} D i v i s i o n}$ of Gastroenterology and Hepatology, University of Nebraska Medical Center, Omaha, NE (Neil Bhogal); ${ }^{\text {h}}$ Department of Biostatistics, University of Nebraska Medical Center, Omaha, NE (Harlan Sayles); ${ }^{i}$ Division of Digestive Diseases and Nutrition, University of South Florida Morsani College of Medicine, Tampa, FL

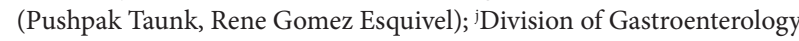
and Hepatology, University of Utah School of Medicine, Salt Lake City, UT (Stephanie McDonough, Douglas G. Adler), USA co-author. The bibliographic section of the selected articles was manually searched for additional relevant articles, as well as systematic and narrative articles on the topic.

\section{Study selection}

In this meta-analysis, we included studies that evaluated the safety of GA and MAC for patients undergoing ERCP. Studies were included irrespectively of inpatient/outpatient setting, geography, abstract/manuscript status, as long as they provided relevant data for the analysis.

The following were our exclusion criteria: (1) studies with sample size $<10$ patients; (2) studies performed in a pediatric population (age <18 years); (3) MAC/GA for procedures other than ERCP; and (4) studies not published in the English language. In cases of multiple publications from the same cohort and/or overlapping cohorts, data from the most recent and/or most appropriate comprehensive report were included.

\section{Data abstraction and quality assessment}

Data on study-related outcomes in the individual studies were abstracted onto a standardized form by at least 3 authors $(\mathrm{BD}, \mathrm{AD}$, $\mathrm{JS})$, and 2 authors (BD, $\mathrm{AD}$ ) did the quality scoring independently.

For randomized trials and case-control studies, the data collected were recorded as number of reported events (n) of total number of patients $(\mathrm{N})$ from each study. The collected data were treated as in single-group cohort studies, and we used the Newcastle-Ottawa scale for cohort and case-control studies to assess the quality of studies [6]. This quality score consisted of 8 questions, the details of which are provided in Table 1.

Quality assessment for randomized controlled trials was performed using the Jadad (Oxford) scale (Table 1). A maximum of 5 points could be awarded to a study on the basis of randomization, blinding and withdrawals from the study. A score of $\leq 3$ was defined as a poor quality study [7].

\section{Outcomes assessed}

The collected data were matched between the groups (general and MAC) before statistical analysis. This model of comparison is indirect, but the approach is comparable to a retrospective case-control study with matched groups.

The primary outcome was to compare the overall rate of adverse events of GA vs. MAC in patients undergoing ERCP. Comparisons between the 2 groups were also performed to evaluate the rate of individual side effects, including, but not limited to, hypoxia, arrhythmias, hypotension, aspiration and other sedation-related adverse events (the data were extracted directly, as reported by the study). The secondary outcome was to compare the total duration of the procedure, recovery time, ERCP cannulation rates, and the rate of conversion of MAC to GA. A subgroup analysis was performed for randomized controlled trials (RCT) and observational studies in the MAC group. 
Table 1 Quality assessment of the study with Newcastle-Ottawa and Jadad (Oxford) scales

\begin{tabular}{|c|c|c|c|c|c|c|}
\hline \multirow{2}{*}{ Author [Ref.] } & \multirow{2}{*}{ Year } & \multirow{2}{*}{ Type of study } & \multirow{2}{*}{ Quality } & \multicolumn{3}{|c|}{ Newcastle-Ottawa scale } \\
\hline & & & & Selection & Comparability & Outcome \\
\hline Patel [29] & 2018 & case control & high & $* * *$ & * & $* * *$ \\
\hline Jokelainen [11] & 2018 & case control & high & $* * *$ & $* *$ & $* *$ \\
\hline Kamani [26] & 2018 & cohort & high & $* * *$ & $*$ & $* * *$ \\
\hline El-Sherif [23] & 2018 & cohort & high & $* * *$ & * & $* * *$ \\
\hline Antoury [20] & 2017 & case control & high & $* * *$ & $*$ & $* * *$ \\
\hline Yang [32] & 2016 & cohort & medium & $* *$ & $*$ & $* * *$ \\
\hline Yuen [33] & 2016 & cohort & high & $* * *$ & * & $* *$ \\
\hline Joshi [25] & 2015 & cohort & high & $* * *$ & $*$ & $* * *$ \\
\hline Khoi [27] & 2015 & cohort & medium & $* *$ & $*$ & $* * *$ \\
\hline Patel [28] & 2014 & cohort & medium & $* *$ & * & $* *$ \\
\hline Sorser [29] & 2014 & case control & high & $* * *$ & $* *$ & $* * *$ \\
\hline Barnett [21] & 2013 & case control & high & $* * *$ & $* *$ & $* * *$ \\
\hline Goudra [24] & 2013 & cohort & medium & $* *$ & $*$ & $* * *$ \\
\hline Amornyotin [19] & 2004 & case control & high & $* * *$ & * & $* * *$ \\
\hline Fanti [9] & 2004 & cohort & high & $* * *$ & $*$ & $* * *$ \\
\hline $\begin{array}{l}\text { Raymonodos } \\
{[30]}\end{array}$ & 2002 & case control & high & $* * *$ & * & $* * *$ \\
\hline \multirow[t]{3}{*}{ Cocking [22] } & 2000 & cohort & medium & ** & * & $* *$ \\
\hline & & & & \multicolumn{3}{|c|}{ Jadad scale } \\
\hline & & & & Randomization & Blinding & Attrition \\
\hline Smith [10] & 2019 & RCT & high & 2 & 0 & 1 \\
\hline Amornyotin [34] & 2011 & RCT & low & 1 & 0 & 1 \\
\hline Jung [12] & 2000 & RCT & low & 1 & 0 & 1 \\
\hline Wehrmann [8] & 1999 & RCT & high & 2 & 0 & 1 \\
\hline
\end{tabular}

RCT, randomized controlled trial

Technical success was defined as successful cannulation in ERCP. The duration of the procedure was defined in only one study, as the time interval from insertion to final withdrawal of the endoscope [8]. Five studies defined the recovery time using different scoring systems: Aldrete score [9,10], Schultz score [11], post-anesthesia recovery score [8], and Seward score [12]. The other studies did not define recovery time.

\section{Statistical analysis}

We used meta-analysis techniques to calculate the pooled estimates in each case, following the methods suggested by DerSimonian and Laird using the random-effects model [13]. When the incidence of an outcome was zero in a study, a continuity correction of 0.5 was added to the number of incident cases before statistical analysis [14]. We assessed heterogeneity between study-specific estimates using the Cochran Q statistical test for heterogeneity and the $I^{2}$ statistic $[15,16]$, for which values of $<30 \%, 30-60 \%, 61-75 \%$ and $>75 \%$ were suggestive of low, moderate, substantial and considerable heterogeneity, respectively [17]. Publication bias was ascertained, qualitatively, by visual inspection of a funnel plot, and quantitatively, by the Egger test [18]. All analyses were performed using STATA v16.1 software (StataCorp, LLC College Station, TX).

\section{Results}

\section{Search results and population characteristics}

From an initial pool of 457 studies, 21 studies reported on the use of GA and MAC in patients undergoing ERCP [8-12,19-34]. Overall, 10 studies provided data on ERCP with GA [10,19- 
23,29-31,33], and 17 studies on ERCP with MAC for our analysis [9-12,20,21,24-34]. Six studies were included in both the ERCP and MAC groups [10,20,21,29,31,33]. The schematic diagram of study selection as per PRISMA guidelines is illustrated in Fig. 1.

The mean patient age was 60.4 and 58.92 years in the GA and MAC groups, respectively, with a predominantly male population in the GA group (51.9\% reported in 9 studies). Patient demographic characteristics are described in Table 2.

\section{Characteristics and quality of included studies}

Eleven studies were prospective and the rest were retrospective in nature. One study was multicenter and the rest were single-center. None were population-based. All studies reported adequately on the clinical outcomes, assessments, and factors of interest. Overall, 14 studies were considered of high quality, 5 were of medium quality, and 2 were low-quality studies. The detailed assessment of study quality is shown in Table 1.

\section{Meta-analysis outcomes}

A total of 11,592 patients were included in the analysis. ERCP was performed under GA and MAC in 3062 and 8530 patients, respectively. The overall adverse event rates among patients undergoing ERCP with GA vs. MAC were similar: $12.76 \%$, $95 \%$ confidence interval $[\mathrm{CI}] 5.80-21.73 ; I^{2}=95 \%$ vs. $12.08 \%$, 95\%CI 5.38-20.89; $I^{2}=99 \%$, respectively $(\mathrm{P}=0.956)$. Adverse events are described in Table 3 (Fig. 2).

Hypoxia was more prevalent in the MAC group (1.88\%, $95 \% \mathrm{CI}=0.04-5.48 ; I^{2}=99.11 \%$ vs. $0.01 \%, 95 \% \mathrm{CI} \quad 0.00-0.55$; $\left.I^{2}=58.87 \% ; \mathrm{P}=0.067\right)$ whereas hypotension was more prevalent in the GA group $\left(7.01 \%, 95 \%\right.$ CI $1.62-15.19 ; I^{2}=99.25 \%$ vs. 4.74\%, 95\%CI 2.18-8.11; $\left.I^{2}=97.84 \% ; \mathrm{P}=0.309\right)$. The P-value was not statistically significant for either hypoxia or hypotension. Overall pooled rates of arrhythmias were similar between GA $\left(0.09 \%, 95 \% \mathrm{CI} 0.00-0.84 ; I^{2}=60.13 \%\right)$ and MAC $(0.08 \%, 95 \% \mathrm{CI}$ $\left.0.00-0.36 ; I^{2}=75.17 \%\right)$ groups with $\mathrm{P}=0.40$. Overall pooled rates for aspiration pneumonia, post-ERCP pancreatitis (PEP) and other sedation-related adverse events were similar between the 2 groups, with non-significant $\mathrm{P}$-values. Forest plots for overall

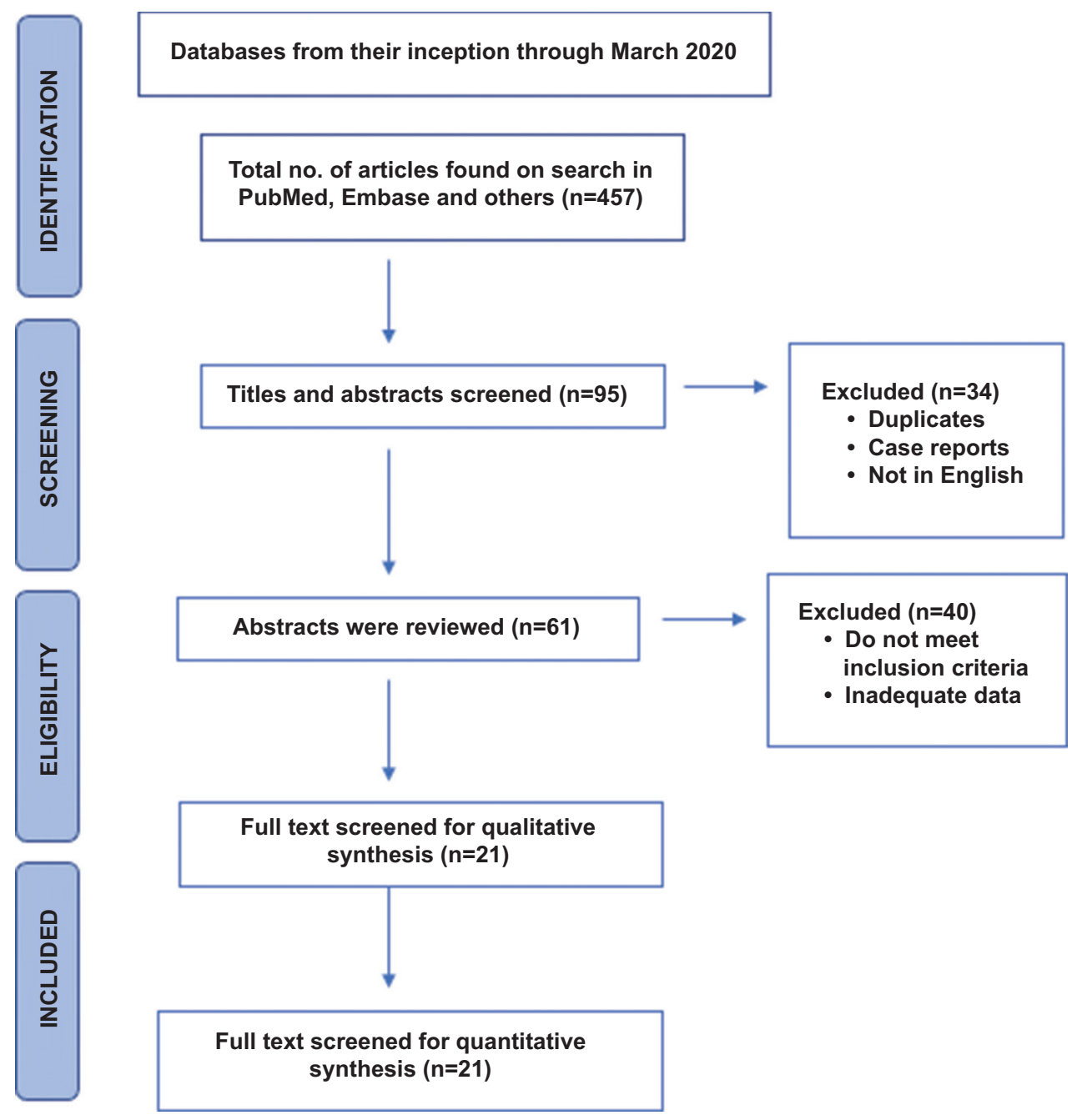

Figure 1 Study selection process in accordance with preferred reporting items for systematic reviews and meta-analysis statement 
Table 2 Description of 21 studies used in the final analysis

\begin{tabular}{|c|c|c|c|c|c|c|c|c|}
\hline Author [Ref.] & Study year & Study type & $\begin{array}{l}\text { Single-center } \\
\text { or multicenter }\end{array}$ & $\begin{array}{l}\text { Abstract or } \\
\text { Manuscript }\end{array}$ & $\begin{array}{l}\text { No. of } \\
\text { patients }\end{array}$ & Mean age & Male & Female \\
\hline \multicolumn{9}{|c|}{ Monitored anesthesia care } \\
\hline Smith [10] & 2019 & Prospective & single & manuscript & 99 & 61.3 & 57 & 42 \\
\hline Patel [29] & 2018 & Prospective & single & abstract & 366 & 60.5 & 218 & 148 \\
\hline Jokelainen [11] & 2018 & Prospective & single & manuscript & 133 & 58 & 77 & 56 \\
\hline Kamani [26] & 2018 & Retrospective & single & manuscript & 550 & 50.85 & 197 & 353 \\
\hline Antoury [20] & 2017 & Retrospective & single & abstract & 50 & 62 & 11 & 39 \\
\hline Yang [32] & 2016 & Retrospective & single & manuscript & 3041 & 58.2 & 1399 & 1642 \\
\hline Yuen [33] & 2016 & Retrospective & single & manuscript & 185 & 57.3 & 106 & 79 \\
\hline Khoi [27] & 2015 & Retrospective & single & manuscript & 552 & 60 & 283 & 269 \\
\hline Joshi [25] & 2015 & Prospective & single & manuscript & 744 & 63 & 333 & 296 \\
\hline Patel [28] & 2014 & RetWrospective & single & abstract & 829 & 45.1 & 249 & 580 \\
\hline Sorser [31] & 2014 & Retrospective & multi & manuscript & 367 & - & - & - \\
\hline Barnett [21] & 2013 & Prospective & single & manuscript & 393 & 63.4 & 183 & 210 \\
\hline Goudra [24] & 2013 & Retrospective & single & manuscript & 653 & 60.5 & 420 & 233 \\
\hline Amornyotin [34] & 2011 & Prospective & single & manuscript & 86 & 58.2 & 41 & 45 \\
\hline Fanti [9] & 2004 & Prospective & single & manuscript & 205 & 63 & 97 & 108 \\
\hline Jung [12] & 2000 & Prospective & single & manuscript & 80 & 62.5 & 32 & 48 \\
\hline Wehrmann [8] & 1999 & Prospective & single & manuscript & 197 & - & - & - \\
\hline \multicolumn{9}{|l|}{ General anesthesia } \\
\hline Smith [10] & 2019 & Prospective & single & manuscript & 101 & 60.9 & 64 & 37 \\
\hline Patel [29] & 2018 & Prospective & single & abstract & 136 & 52.3 & 71 & 65 \\
\hline El-Sherif [23] & 2018 & Prospective & single & manuscript & 67 & 55.8 & 27 & 37 \\
\hline Antoury [20] & 2017 & Retrospective & single & abstract & 50 & 70 & 14 & 36 \\
\hline Yuen [33] & 2016 & Retrospective & single & manuscript & 21 & 55.7 & 12 & 9 \\
\hline Sorser [31] & 2014 & Retrospective & multi & manuscript & 283 & - & - & - \\
\hline Barnett [21] & 2013 & Prospective & single & manuscript & 45 & 65.9 & 25 & 20 \\
\hline Amornyotin [19] & 2004 & Retrospective & single & manuscript & 2144 & 57.2 & 1106 & 1108 \\
\hline Raymonodos [30] & 2002 & Retrospective & single & manuscript & 190 & 42 & 118 & 72 \\
\hline Cocking [22] & 2000 & Prospective & single & manuscript & 25 & 84.2 & 8 & 17 \\
\hline
\end{tabular}

adverse events and individual adverse events are provided in Supplementary Figs. 1-5.

The secondary outcome was to compare the total duration of the procedure, recovery time, ERCP cannulation rates, and the need to convert from MAC to GA. Technical success was comparable between MAC (97.17\%, 95\%CI 95.25-98.65; $\left.I^{2}=78.6 \%\right)$ and GA (95.29\%, 95\%CI 90.76-98.51; $\left.I^{2}=73.04 \%\right)$ groups $(\mathrm{P}=0.232)$ (Supplementary Fig. 6). The recovery time and duration of the procedure were reported in 8 and 11 studies, respectively. Although, the mean duration of the procedure was longer in the MAC group (47 vs. $33 \mathrm{~min}$ ) mean recovery time was shorter when compared to the GA group ( 47 vs. $59 \mathrm{~min}$ ). A total of $1.7 \%$ of patients in MAC group were converted to $\mathrm{GA}$, with a range varying from 0.4 $10.1 \%$.

On subgroup analysis of the MAC studies, RCT $(n=4)$ had similar rates of adverse events (12.93\%, 95\%CI 4.25-25.28; $\left.I^{2}=99.36 \%\right)$ compared to observational studies $(12.69 \%, 95 \% \mathrm{CI}$ 2.39-29.02; $\left.I^{2}=97.92 \%\right)$, with a P-value of 0.989 . Technical success was also similar in RCT as compared to observational studies $\left(97.98 \%\right.$, 95\%CI 95.09-99.71; $I^{2}=82.12 \%$ vs. $96.41 \%$, 95\%CI 93.25-98.67; $I^{2}=75.85 \%$; $\left.\mathrm{P}=0.385\right)$. Individual adverse events were also comparable. No subgroup analysis was possible for the GA studies as there were insufficient RCTs in this group.

\section{Validation of meta-analysis results}

\section{Sensitivity analysis}

To assess whether any one study had a dominant effect on the meta-analysis, we excluded one study at a time and analyzed its effect on the main summary estimate. On this analysis, no single study significantly affected the outcome or the heterogeneity.

\section{Heterogeneity}

We assessed the dispersion of the calculated rates using $I^{2}$ percentage values. The $I^{2}$ tells us what proportion of the dispersion is true vs. chance [15]. Overall pooled rates for sedation-related adverse events, individual adverse events and technical success showed substantial to considerable heterogeneity.

\section{Publication bias}

Based on visual inspection of the funnel plot, there seemed to be greater possible publication bias in the MAC compared to the GA group. Based on quantitative analysis via the Egger regression test, the statistical 2-tailed P-value was 0.004 
Table 3 Adverse events in monitored anesthesia care and general anesthesia

\begin{tabular}{|c|c|c|c|c|c|c|c|c|}
\hline Author [Ref.] & Study year & $\begin{array}{c}\text { Total adverse } \\
\text { effects }\end{array}$ & Hypotension & Hypoxia & Arrhythmia & $\begin{array}{l}\text { Aspiration } \\
\text { pneumonia }\end{array}$ & PEP & Others \\
\hline \multicolumn{9}{|c|}{ Monitored anesthesia care } \\
\hline Smith [10] & 2019 & 51 & 9 & 19 & 0 & 0 & 1 & 23 \\
\hline Patel [29] & 2018 & 9 & 0 & 0 & 0 & 0 & 10 & 9 \\
\hline Jokelainen [11] & 2018 & 17 & 15 & 1 & 1 & 0 & 0 & 0 \\
\hline Kamani [26] & 2018 & 2 & 0 & 0 & 0 & 0 & 0 & 2 \\
\hline Antoury [20] & 2017 & 14 & 10 & 2 & 2 & 0 & 0 & 0 \\
\hline Yang [32] & 2016 & 872 & 20 & 843 & 0 & 6 & 0 & 3 \\
\hline Yuen [33] & 2016 & 93 & 38 & 15 & 0 & 0 & 0 & 40 \\
\hline Khoi [27] & 2015 & 166 & 165 & 1 & 0 & 0 & 0 & 0 \\
\hline Joshi [25] & 2015 & 8 & 3 & 2 & 1 & 1 & 0 & 2 \\
\hline Patel [28] & 2014 & 1 & 0 & 1 & 0 & 0 & 0 & 0 \\
\hline Sorser [31] & 2014 & 55 & 0 & 17 & 5 & 0 & 19 & 23 \\
\hline Barnett [21] & 2013 & 85 & 16 & 59 & 10 & 0 & 0 & 0 \\
\hline Goudra [24] & 2013 & 3 & 0 & 3 & 0 & 0 & 0 & 0 \\
\hline Amornyotin [34] & 2011 & 26 & 18 & 1 & 5 & 0 & 0 & 2 \\
\hline Fanti [9] & 2004 & 4 & 0 & 4 & 0 & 0 & 0 & 0 \\
\hline Jung [12] & 2000 & 3 & 1 & 2 & 0 & 0 & 0 & 0 \\
\hline Wehrmann [8] & 1999 & 26 & 9 & 10 & 0 & 0 & 13 & 7 \\
\hline \multicolumn{9}{|l|}{ General anesthesia } \\
\hline Smith [10] & 2019 & 10 & 10 & 0 & 0 & 0 & 0 & 0 \\
\hline Patel [29] & 2018 & 7 & 0 & 0 & 0 & 0 & 7 & 7 \\
\hline El-Sherif [23] & 2018 & 0 & 0 & 0 & 0 & 0 & 0 & 0 \\
\hline Antoury [20] & 2017 & 28 & 22 & 1 & 5 & 0 & 0 & 0 \\
\hline Yuen [33] & 2016 & 17 & 10 & 0 & 0 & 0 & 0 & 7 \\
\hline Sorser [31] & 2014 & 9 & 0 & 1 & 0 & 0 & 0 & 8 \\
\hline Barnett [21] & 2013 & 13 & 8 & 3 & 2 & 0 & 0 & 0 \\
\hline Amornyotin [19] & 2004 & 194 & 171 & 0 & 8 & 0 & 0 & 15 \\
\hline Raymonodos [30] & 2002 & 1 & 0 & 0 & 0 & 0 & 0 & 1 \\
\hline Cocking [22] & 2000 & 0 & 0 & 0 & 0 & 0 & 0 & 0 \\
\hline
\end{tabular}

PEP, post-endoscopic retrograde cholangiopancreatography pancreatitis

for the MAC group and 0.605 for the GA group. Refer to Supplementary Figs. 7 and 8 for the funnel plots.

\section{Discussion}

The choice between MAC and GA as a means of sedation for patients undergoing ERCP depends upon many factors, including the patient's acuity, operator experience, institutional practices, and the preferences and experience of the endoscopists and the anesthesiologist [35].

High-risk features that may result in sedation-related adverse events include having an American Society of Anesthesiologists (ASA) class greater than 3, obstructive sleep apnea, male sex, body mass index (BMI) greater than $30 \mathrm{~kg} / \mathrm{m}^{2}$, presence of abdominal ascites prior to ERCP, Mallampati class 4, Cotton grade greater than 3 , and heavy alcohol use ( $>3$ drinks for men and $>2$ drinks for women) $[9,10,12,19,21,25,27,32,35,36]$. The overall adverse event rate was comparable between MAC and GA $(\mathrm{P}=0.956)$. The subgroup analysis of the individual adverse events showed hypoxia was more common with MAC than GA, but the difference was not statistically significant $(\mathrm{P}=0.067)$. Fanti et al reported a similar rate $(1.9 \%)$ of hypoxiarelated adverse events. Yang et al reported hypoxia in $28 \%$ of their patients when MAC was utilized for ERCP, but it resolved with airway manipulation. Of these, only $0.1 \%$ of cases were terminated because of refractory laryngospasm [32]. On the other hand, Goudra et al did not describe a significant number of hypoxia-related events with MAC, and this was attributed to the low threshold of desaturation ( $<95 \%$ oxygen saturation) compared to $<90 \%$ oxygen saturation in other studies. The threshold of $<95 \%$ allowed earlier supplementation of oxygen through airway conduits to avoid episodes of hypoxia [24].

Hypotension was more prevalent among patients undergoing GA vs. MAC, but the difference was not statistically significant $(\mathrm{P}=0.309)$. Amorynotin et al reported hypotension in $8.8 \%$ of their patients with GA, mostly seen after rapid propofol injection, and it was corrected with fluids and vasopressors [19]. Khoi et al reported that $29.9 \%$ of their 552 patients experienced hypotension with MAC, and this was attributed to older age (67.7 years) and a longer duration of anesthesia [27]. The overall pooled rates of arrhythmias were low and comparable in GA vs. MAC with a nonsignificant P-value of 0.40 .

The pooled rates for aspiration pneumonia $(\mathrm{P}=0.172), \mathrm{PEP}$ $(\mathrm{P}=0.867)$, and other sedation-related adverse events $(\mathrm{P}=0.79)$ in our study were similar between the 2 groups. No cases of aspiration pneumonia were reported in the GA group, but 7 were reported in the MAC group [25,32]. Yang et al reported that 6 of their 7 cases of aspiration pneumonia were in the MAC group. This was seen 


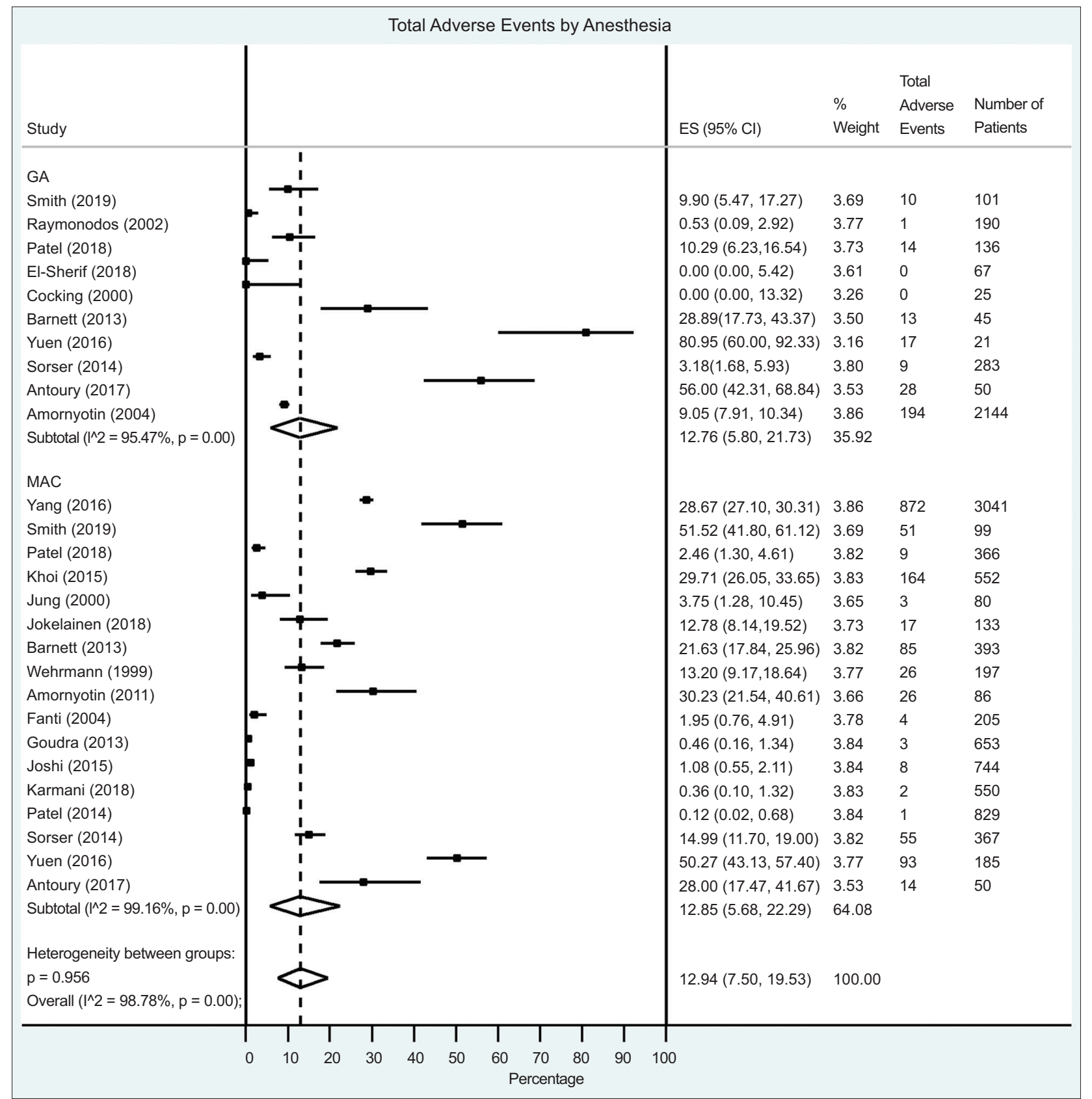

Figure 2 Forest plot showing overall adverse events of endoscopic retrograde cholangiopancreatography under MAC and GA MAC, monitored anesthesia care; GA, general anesthesia; ES, effect size; CI, confidence interval

in individuals converted to GA as a measure to prevent aspiration, since there were gastric contents seen on endoscopy [32].

Conversion from MAC to GA occurred in $1.7 \%$ of patients, ranging from $0.4-10.1 \%$. Yang et al reported that $1.6 \%$ of patients underwent conversion [32]. In our meta-analysis, the highest conversion rate $(10.1 \%)$ was seen in the study by Smith et al. This was the first prospective RCT to compare GA vs. MAC. The reasons for conversion in our meta-analysis are multifactorial and include visualization of gastric contents during endoscopy (aspiration prophylaxis), ASA class $>3$, respiratory compromise refractory to airway maneuvers, chronic obstructive pulmonary disease, BMI $>30 \mathrm{~kg} / \mathrm{m}^{2}$, and agitation/restlessness despite maximum sedation $[10,21,24,26,32,35]$.

ERCP cannulation rates were comparable between MAC and $\mathrm{GA}$ at $97.17 \%(95 \% \mathrm{CI} 95.25-98.65)$ vs. $95.29 \%$ (95\%CI 90.76-98.51), respectively $(\mathrm{P}=0.232)$. In a study by Raymondos et al, MAC was associated with more unsuccessful ERCP procedures as compared to GA ( $14 \%$ vs. $7 \%$; $\mathrm{P}=0.012)$ [30]. This was attributed to the endoscopist's level of experience, as the ERCP procedures under GA were performed by more experienced endoscopists, whereas ERCP with MAC was performed by a less experienced endoscopist [30]. 
The mean procedural duration was longer in the MAC group ( 47 vs. $33 \mathrm{~min}$ ), but the mean recovery time was shorter when compared to the GA group (47 vs. $59 \mathrm{~min}$ ). Fanti et al attributed a long procedure time with MAC to the complexity of the ERCPs [9]. The shorter recovery times in the MAC group were associated with propofol utilization, as the GA group utilized several anesthetic agents including paralytics, benzodiazepines, opiates and/or propofol $[8,9,20,29,30,33,34]$. It has been reported that shorter recovery times are associated with propofol utilization compared to benzodiazepines/opiates [9,23,33,35].

There are several limitations in our study. Heterogeneity was considerable to substantial in our meta-analysis in regard to the overall pooled rates for sedation-related adverse events, individual adverse events and technical success. This may be due to the indications for the procedure, choice of sedation, anesthesia provider, endoscopist, patient population, or endoscopy technique. A definition of procedure time was only provided in one study. Defining the procedure time as patient in room to scope out vs. scope in to scope out, may factor in the time it takes to intubate and could provide a useful measure. Many of our studies were retrospective and did not undergo randomization, which introduces selection bias. Most studies were conducted at single tertiary referral centers, so results may be difficult to generalize. The results from a high-volume endoscopy center may not be generalizable to a low-volume endoscopy center. Another limitation was the presence of publication bias, as measured by the Eggers regression test. Lastly, the studies included did not compare the healthcare costs associated with either of the methods for sedation.

The results of our meta-analysis show that overall adverse events, individual adverse events, and technical success were comparable between the 2 groups. Although not statistically significant, MAC had higher rates of hypoxia, lower rates of hypotension, longer procedure times, and a shorter recovery period as compared to GA.

\section{Summary Box}

\section{What is already known:}

- Endoscopic retrograde cholangiopancreatography (ERCP) is the treatment of choice in pancreaticobiliary diseases

- There is ongoing debate regarding the relative merits and risks of monitored anesthesia care (MAC) vs. general anesthesia (GA) for patients undergoing ERCP

\section{What the new findings are:}

- Sedation-related side effects were similar in the GA and MAC groups

- Although not statistically significant, MAC had higher rates of hypoxia, lower rates of hypotension, longer procedure times, and a shorter recovery period compared to GA

\section{References}

1. Yachimski PS, Ross A. The future of endoscopic retrograde cholangiopancreatography. Gastroenterology 2017;153:338-344.

2. American Society of Anesthesiologists. Continuum of depth of sedation: definition of general anesthesia and levels of sedation/ analgesia. 2019. Available from: https://www.asahq.org/standardsand-guidelines/continuum-of-depth-of-sedation-definition-ofgeneral-anesthesia-and-levels-of-sedationanalgesia [Accessed 2 June 2021].

3. Das S, Ghosh S. Monitored anesthesia care: an overview. J Anaesthesiol Clin Pharmacol 2015;31:27-29.

4. Law R, Cardenas A. General endotracheal anesthesia for ERCP: don't sleep on it! Gastroint Endosc 2019;89:863-864.

5. Moher D, Liberati A, Tetzlaff J, Altman DG; PRISMA Group. Preferred reporting items for systematic reviews and metaanalyses: the PRISMA statement. Ann Intern Med 2009;151:264269, W64.

6. Stang A. Critical evaluation of the Newcastle-Ottawa scale for the assessment of the quality of nonrandomized studies in metaanalyses. Eur J Epidemiol 2010;25:603-605.

7. Jadad AR, Moore RA, Carroll D, et al. Assessing the quality of reports of randomized clinical trials: is blinding necessary? Control Clin Trials 1996;17:1-12.

8. Wehrmann T, Kokabpick S, Lembcke B, Caspary WF, Seifert H. Efficacy and safety of intravenous propofol sedation during routine ERCP: a prospective, controlled study. Gastrointest Endosc 1999;49:677-683.

9. Fanti L, Agostoni M, Casati A, et al. Target-controlled propofol infusion during monitored anesthesia in patients undergoing ERCP. Gastrointest Endosc 2004;60:361-366.

10. Smith ZL, Mullady DK, Lang GD, et al. A randomized controlled trial evaluating general endotracheal anesthesia versus monitored anesthesia care and the incidence of sedation-related adverse events during ERCP in high-risk patients. Gastrointest Endosc 2019;89:855-862.

11. Jokelainen J, Mustonen H, Kylänpää L, Udd M, Lindström O, Pöyhiä R. Assessment of sedation level for endoscopic retrograde cholangiopancreatography - a prospective validation study. Scand J Gastroenterol 2018;53:370-375.

12. Jung M, Hofmann C, Kiesslich R, Brackertz A. Improved sedation in diagnostic and therapeutic ERCP: propofol is an alternative to midazolam. Endoscopy 2000;32:233-238.

13. DerSimonian R, Laird N. Meta-analysis in clinical trials. Control Clin Trials 1986;7:177-188.

14. Sutton AJ, Abrams KR, Jones DR, et al. Methods for meta-analysis in medical research: Wiley Chichester; 2000.

15. Higgins JP, Thompson SG, Deeks JJ, Altman DG. Measuring inconsistency in meta-analyses. BMJ 2003;327:557-560.

16. Kanwal F, White D. "Systematic reviews and meta-analyses" in clinical gastroenterology and hepatology. Clin Gastroenterol Hepatol 2012;10:1184-1186.

17. Guyatt GH, Oxman AD, Kunz R, et al; GRADE Working Group. GRADE guidelines: 7. Rating the quality of evidenceinconsistency. J Clin Epidemiol 2011;64:1294-1302.

18. Easterbrook PJ, Berlin JA, Gopalan R, Matthews DR. Publication bias in clinical research. Lancet 1991;337:867-872.

19. Amornyotin S, Na-pomphet S, Wongwathanyoo T, Chalayonnavin V. Anesthesia for endoscopic retrograde cholangiopancreatography (ERCP) from 1999-2003 in Siriraj Hospital: a retrospective study. J Med Assoc Thai 2004;87:1491-1495.

20. Antoury C, Thakkar S, Dhawan M, Farah K, Kulkarni A. Sa1383 Outcomes of endoscopic retrograde cholangiopancreatography (ERCP) performed under general anesthesia compared to moderate anesthesia care. Gastrointest Endosc 2017;85:AB218.

21. Barnett SR, Berzin T, Sanaka S, Pleskow D, Sawhney M, Chuttani 
R. Deep sedation without intubation for ERCP is appropriate in healthier, non-obese patients. Dig Dis Sci 2013;58:3287-3292.

22. Cocking JB, Ferguson A, Mukherjee SK, Giancola G. Shortacting general anaesthesia facilitates therapeutic ERCP in frail elderly patients with benign extra-hepatic biliary disease. Eur J Gastroenterol Hepatol 2000;12:451-454.

23. El-Sherif Y, Hunt J, Suddle A, et al. Day case 'treat and transfer' ERCP service under general anaesthesia. Frontline Gastroenterol 2018;9:317-322.

24. Goudra BG, Singh PM, Sinha AC. Outpatient endoscopic retrograde cholangiopancreatography: Safety and efficacy of anesthetic management with a natural airway in 653 consecutive procedures. Saudi J Anaesth 2013;7:259-265.

25. Joshi D, Paranandi B, El Sayed G, et al. Experience of propofol sedation in a UK ERCP practice: lessons for service provision. Frontline Gastroenterol 2015;6:32-37.

26. Kamani L, Memon AL, Anwar A. Safety of conscious sedation in patients undergoing endoscopic retrograde cholangio pancreatography. J Coll Physicians Surg Pak 2018;28:950-952.

27. Khoi CS, Wong JJ, Wang HC, Lu CW, Lin TY. Age correlates with hypotension during propofol-based anesthesia for endoscopic retrograde cholangiopancreatography. Acta Anaesthesiol Taiwan 2015;53:131-134.

28. Patel A, Sreenarasimhaiah J, Agrawal D. Mo1369 Safety and efficacy of moderate sedation for ERCPs. Gastrointest Endosc 2012;75:AB403.

29. Patel RJ, Nelsen EM, Akhter A, et al. 939 General anesthesia versus moderate concious sedation: a prospective study on outcomes with selective sedation in ERCP. Gastrointest Endosc 2018;87:AB136-
AB137.

30. Raymondos K, Panning B, Bachem I, Manns MP, Piepenbrock S, Meier PN. Evaluation of endoscopic retrograde cholangiopancreatography under conscious sedation and general anesthesia. Endoscopy 2002;34:721-726.

31. Sorser SA, Fan DS, Tommolino EE, et al. Complications of ERCP in patients undergoing general anesthesia versus MAC. Dig Dis Sci 2014;59:696-697.

32. Yang JF, Farooq P, Zwilling K, Patel D, Siddiqui AA. Efficacy and safety of propofol-mediated sedation for outpatient endoscopic retrograde cholangiopancreatography (ERCP). Dig Dis Sci 2016;61:1686-1691.

33. Yuen VM, Xia HF, Chan YW, Yan X, Irwin MG. Anesthesiologist-managed sedation for endoscopic retrograde cholangiopancreatography: experience at the University of Hong Kong Shenzhen Hospital. Tech Gastrointest Endosc 2016;18:38-41.

34. Amornyotin S, Srikureja W, Chalayonnavin W, Kongphlay S. Dose requirement and complications of diluted and undiluted propofol for deep sedation in endoscopic retrograde cholangiopancreatography. Hepatobiliary Pancreat Dis Int 2011;10:313-318.

35. Smith ZL, Das KK, Kushnir VM. Anesthesia-administered sedation for endoscopic retrograde cholangiopancreatography: monitored anesthesia care or general endotracheal anesthesia? Curr Opin Anaesthesiol 2019;32:531-537.

36. Berzin TM, Sanaka S, Barnett SR, et al. A prospective assessment of sedation-related adverse events and patient and endoscopist satisfaction in ERCP with anesthesiologist-administered sedation. Gastrointest Endosc 2011;73:710-717. 


\section{Supplementary material}

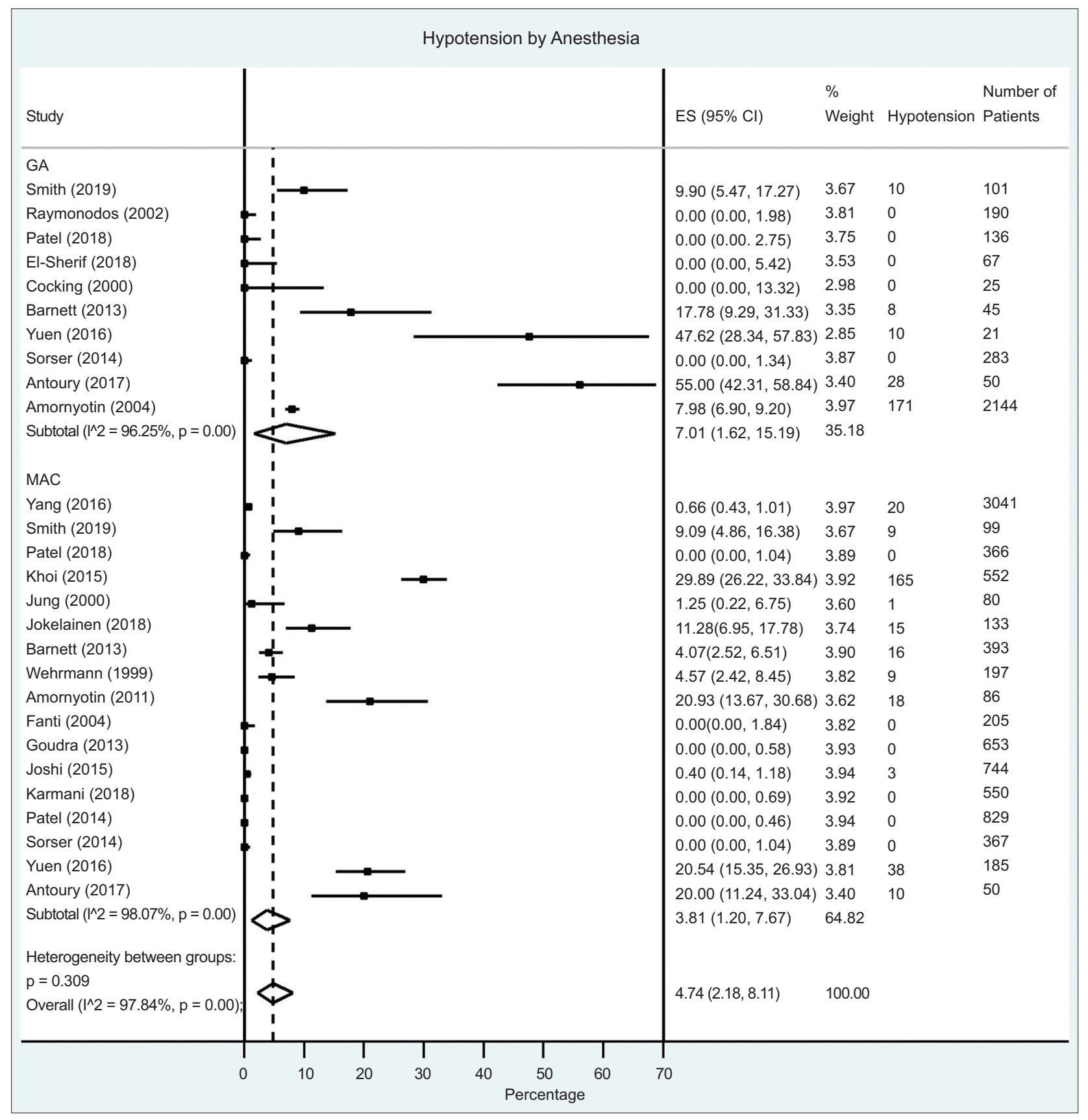

Supplementary Figure 1 Forest plot showing hypotension rates in patients undergoing endoscopic retrograde cholangiopancreatography under MAC and GA

MAC, monitored anesthesia care; GA, general anesthesia; ES, effect size; CI, confidence interval 


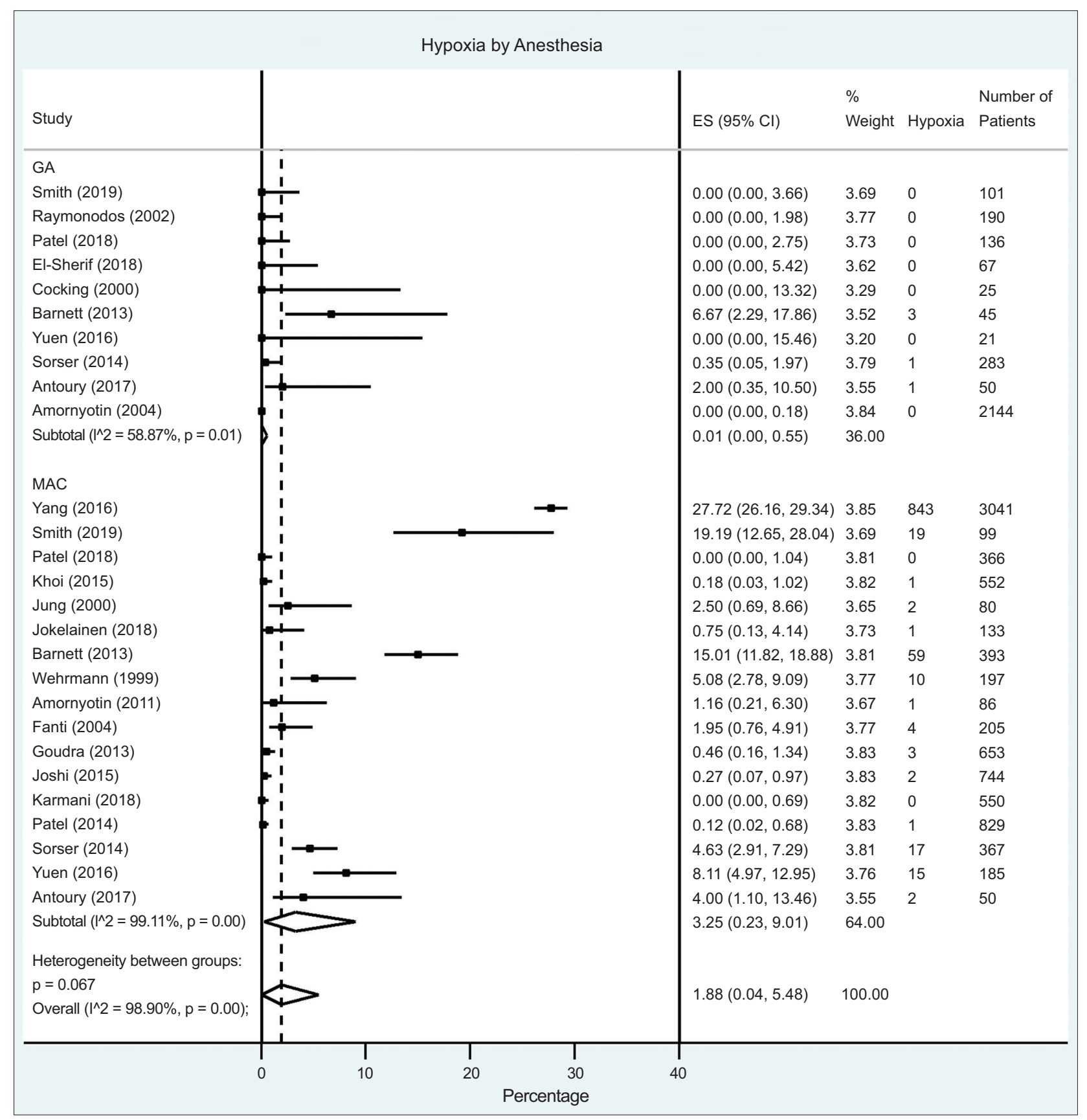

Supplementary Figure 2 Forest plot showing hypoxia rates in patients undergoing endoscopic retrograde cholangiopancreatography under MAC and GA

MAC, monitored anesthesia care; GA, general anesthesia; ES, effect size; CI, confidence interval 


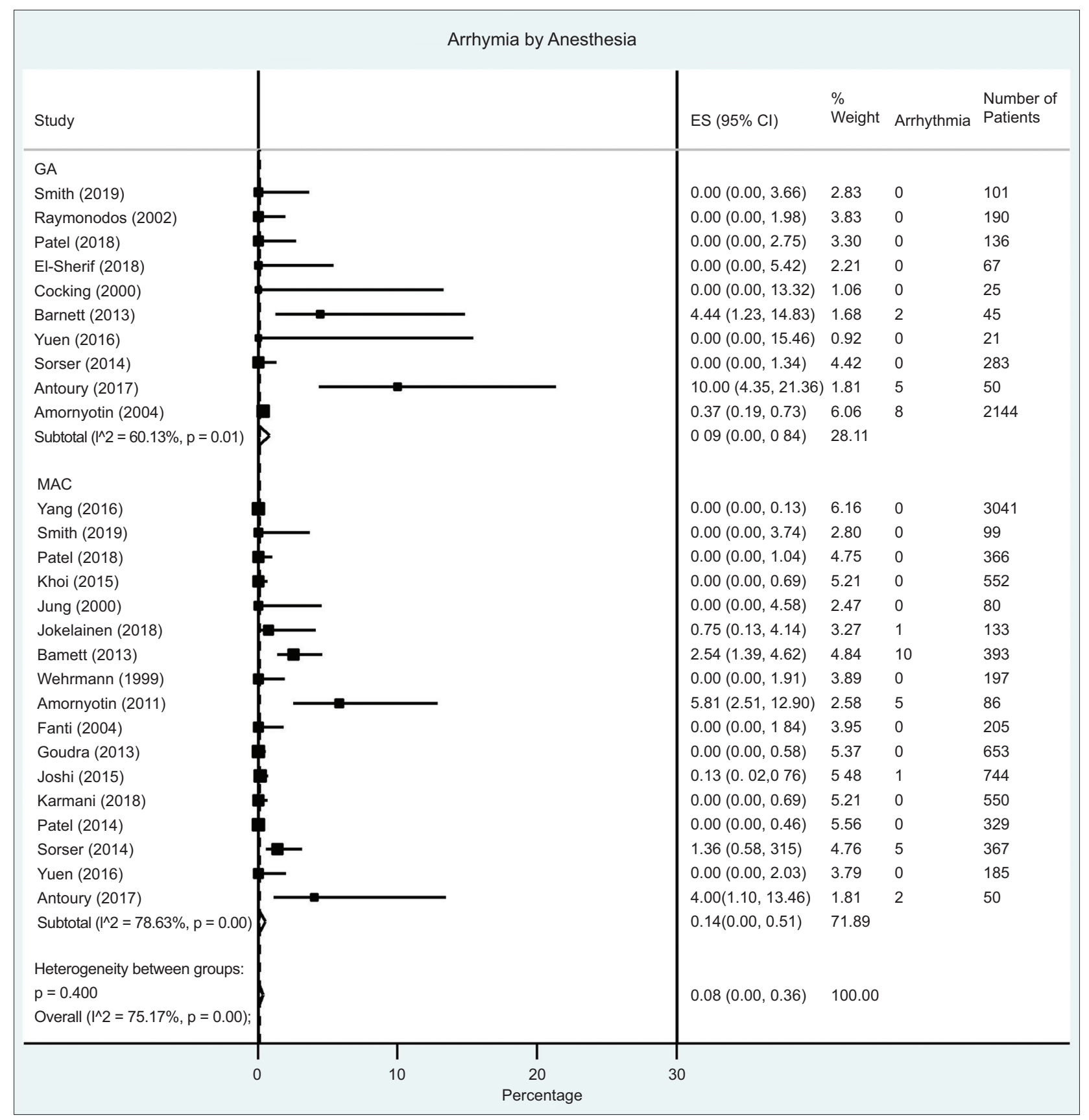

Supplementary Figure 3 Forest plot showing arrhythmia rates in patients undergoing endoscopic retrograde cholangiopancreatography under MAC and GA

MAC, monitored anesthesia care; GA, general anesthesia; ES, effect size; CI, confidence interval 


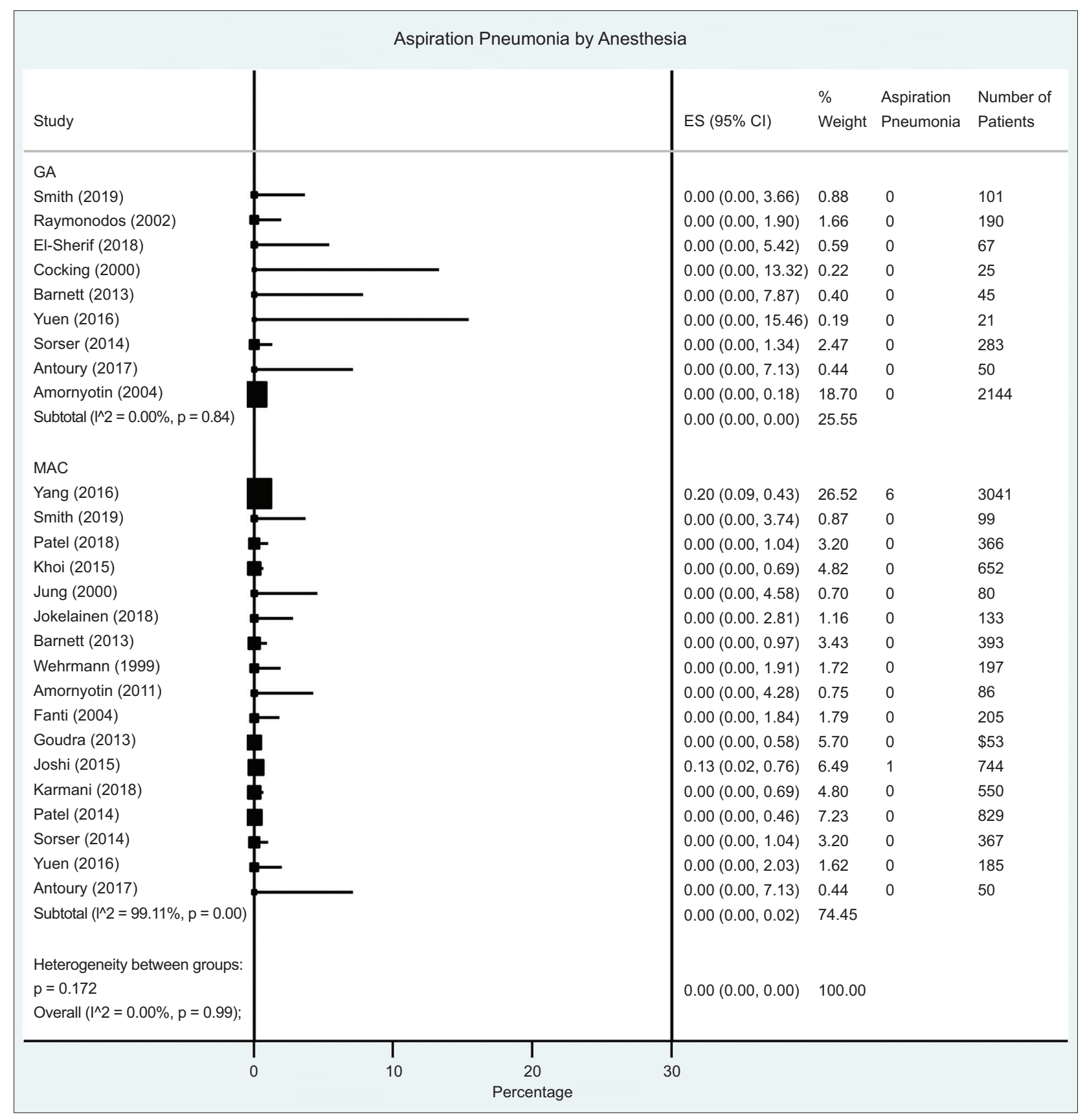

Supplementary Figure 4 Forest plot showing aspiration pneumonia rates in patients undergoing endoscopic retrograde cholangiopancreatography under MAC and GA

$M A C$, monitored anesthesia care; GA, general anesthesia; ES, effect size; CI, confidence interval 


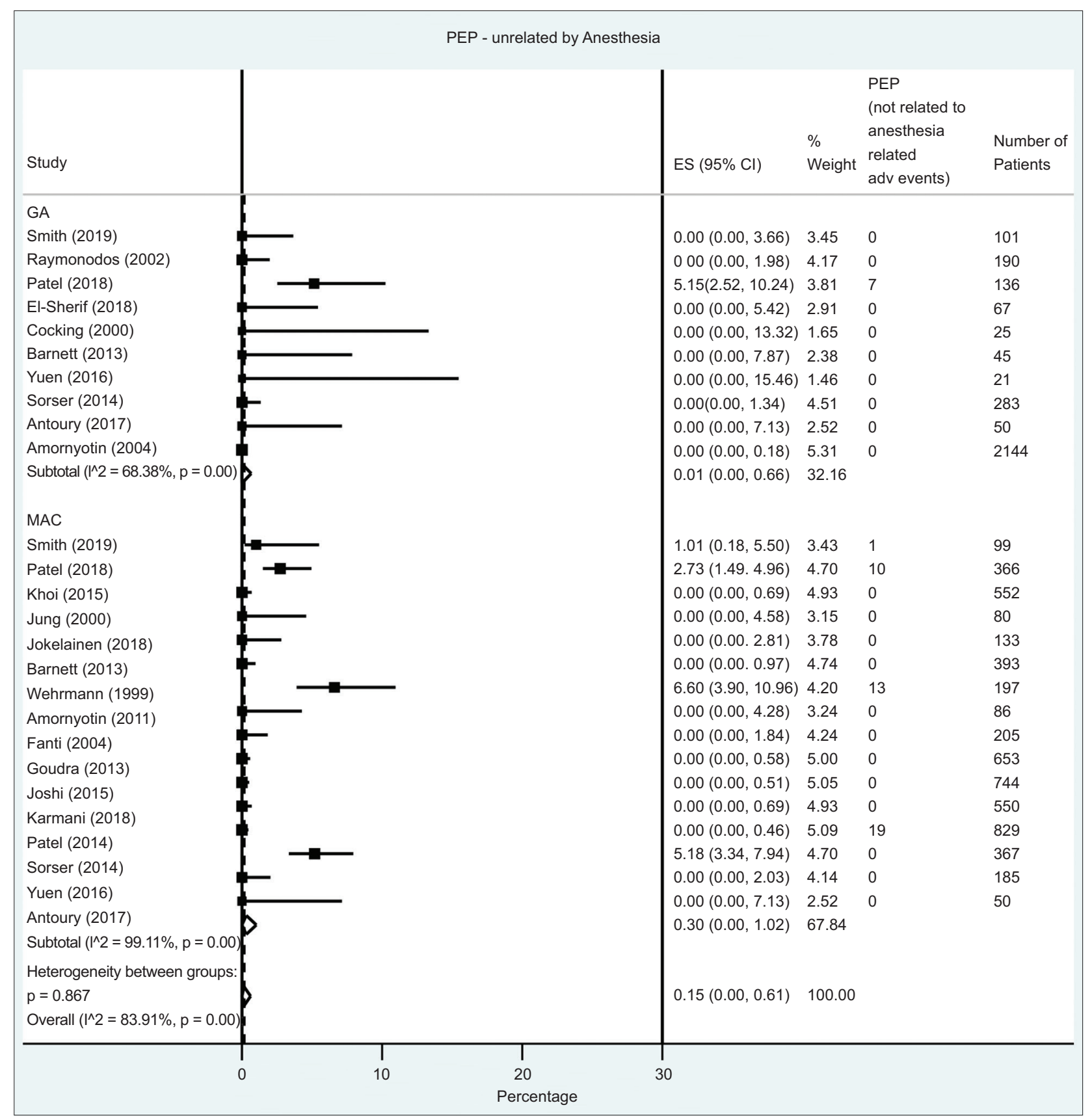

Supplementary Figure 5 Forest plot showing PEP rates in patients undergoing post-endoscopic retrograde cholangiopancreatography under MAC and GA

PEP, pancreatitis; MAC, monitored anesthesia care; GA, general anesthesia; ES, effect size; CI, confidence interval 


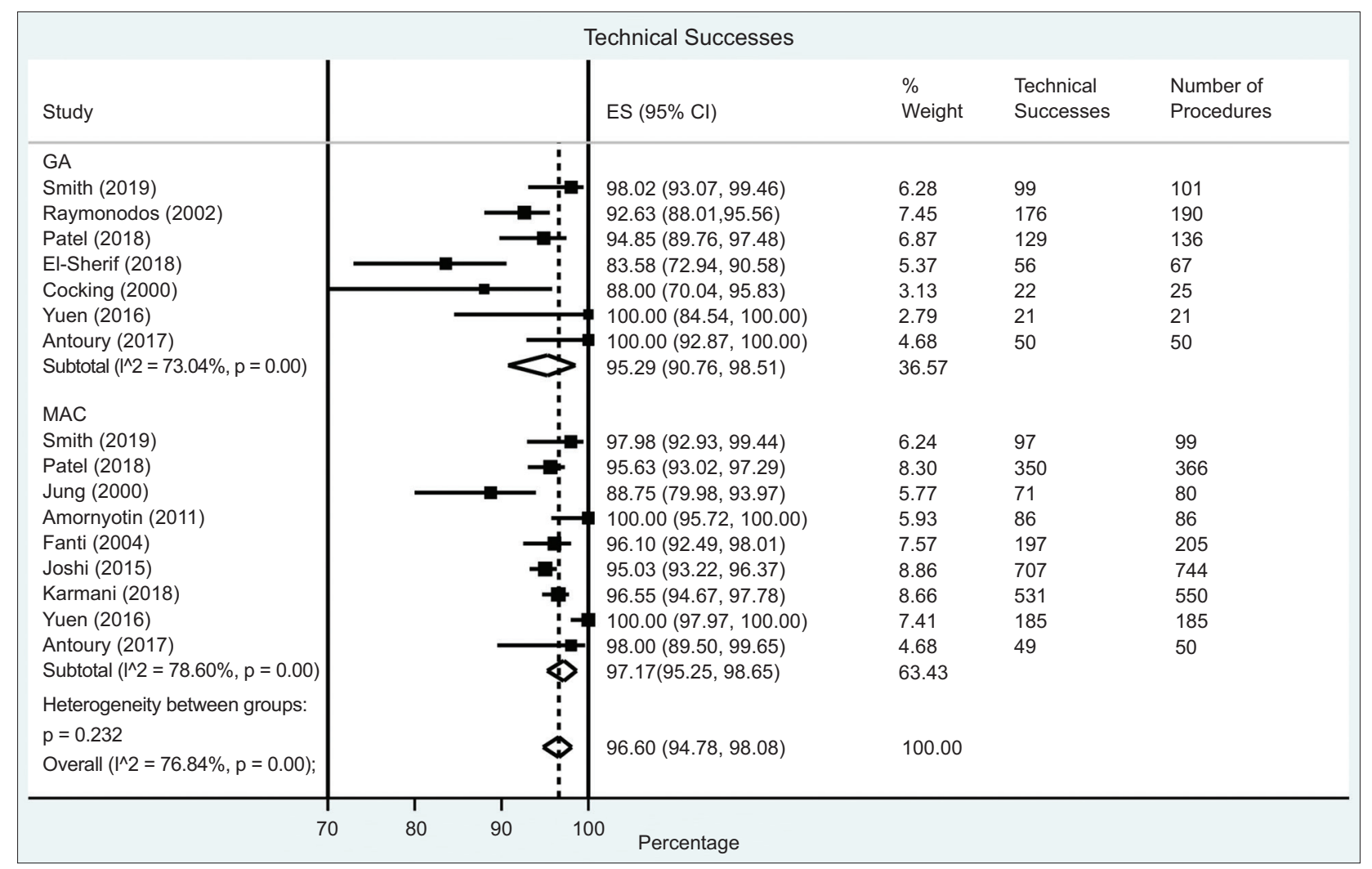

Supplementary Figure 6 Forest plot showing technical success in patients undergoing endoscopic retrograde cholangiopancreatography under MAC and GA

MAC, monitored anesthesia care; GA, general anesthesia; ES, effect size; CI, confidence interval

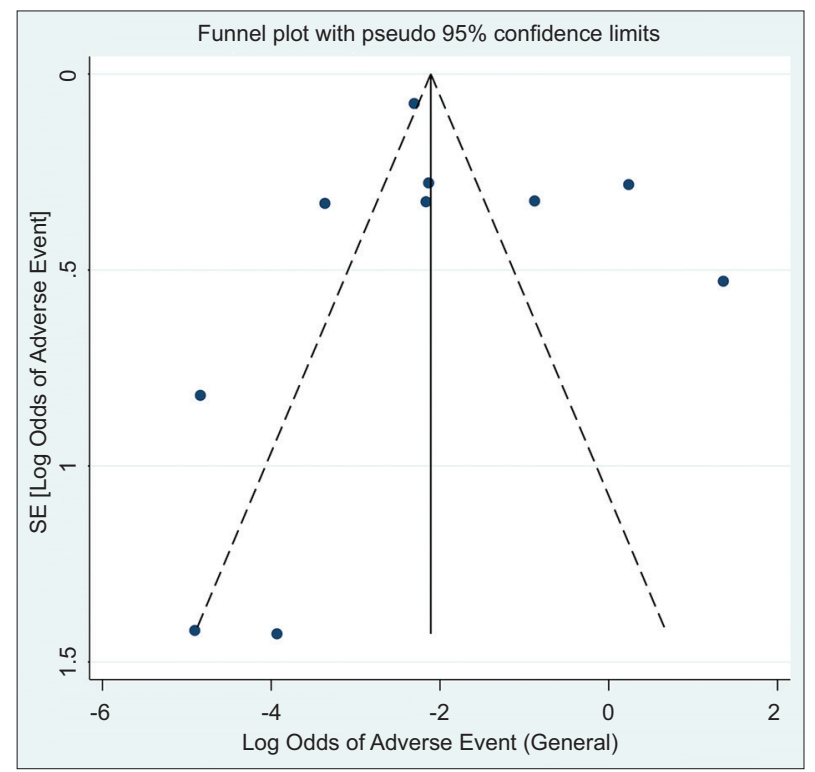

Supplementary Figure 7 Funnel plot for general anesthesia in endoscopic retrograde cholangiopancreatography

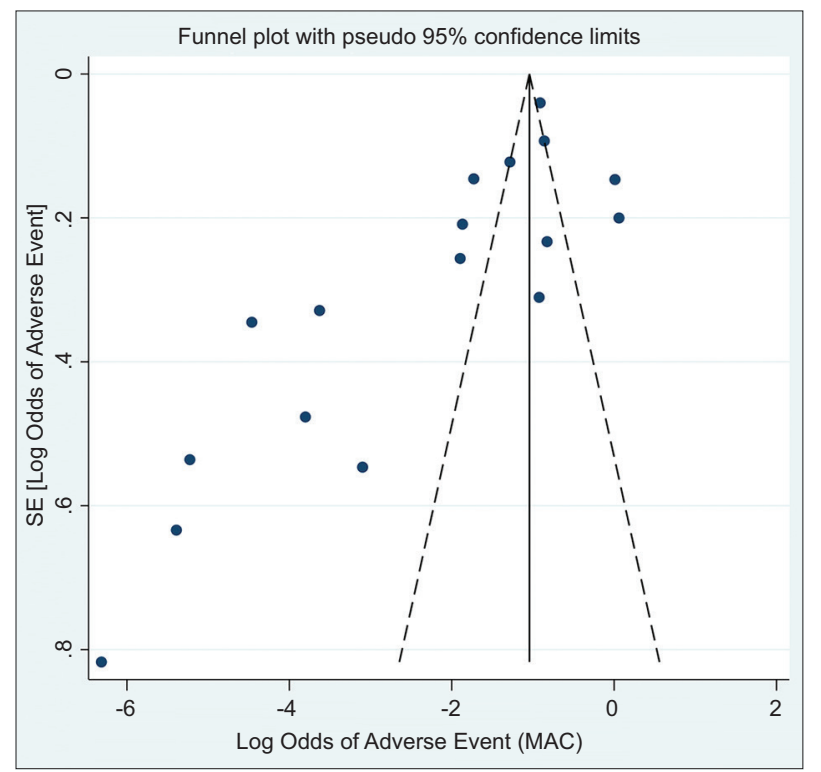

Supplementary Figure 8 Funnel plot for MAC in endoscopic retrograde cholangiopancreatography $M A C$, monitored anesthesia care 